\title{
Anomalous diffusion and anisotropic nonlinear Fokker-Planck equation
}

\author{
E. K. Lenzi ${ }^{1}$, R. S. Mendes ${ }^{1}$ L. C. Malacarne ${ }^{1}$ and L. R. da Silva ${ }^{2}$ \\ 1 Departamento de Física, Universidade Estadual de Maringá, \\ Av. Colombo 5790, 87020-900 Maringá-PR, Brazil \\ 2 International Center for Complex Systems and Departamento de Física, \\ Universidade Federal do Rio Grande do Norte, 59078-970 Natal-RN, Brazil
}

\begin{abstract}
We analyse a bidimensional nonlinear Fokker-Planck equation by considering an anisotropic case, whose diffusion coefficients are $D_{x} \propto|x|^{-\theta}$ and $D_{y} \propto|y|^{-\gamma}$ with $\theta, \gamma \in \mathcal{R}$. In this context, we also investigate two situations with the drift force $\vec{F}(\vec{r}, t)=\left(-k_{x} x,-k_{y} y\right)$. The first one is characterized by $k_{x} / k_{y}=(2+\gamma) /(2+\theta)$ and the second is given by $k_{x}=k$ and $k_{y}=0$. In these cases, we can verify an anomalous behavior induced in different directions by the drift force applied. The found results are exact and exhibit, in terms of the $q$-exponentials, functions which emerge from the Tsallis formalism. The generalization for the $D$-dimensional case is discussed.
\end{abstract}

\section{I - INTRODUCTION}

The existence of the anomalous diffusion and its ubiquity has motived the study of nonlinear Fokker-Planck equation (e.g., $\partial_{t} \rho=\mathcal{D} \nabla^{2} \rho^{\nu}$ ) due to its broadness of applications. In fact, it has been employed in several physical situations such as percolation of gases through porous media $(\nu \geq 2)[1]$, thin saturated regions in porous media $(\nu=2)[2]$, a standard solid-on-solid model for surface growth $(\nu=3)$, thin liquid films spreading under gravity $(\nu=4)[\underline{3}]$, among others [4]. These situations are essentially characterized by correlated anomalous diffusion which has the second moment finite, in contrast with the Levy distribution [5]. In recent works the nonlinear Fokker-Planck equation, present here, has been analysed in one dimension by considering linear force [ 6$]$ and absorption terms [7]. In [ 8 ] a spatial dependence was incorporated in the diffusion coefficient, and in [9] the nonlinear fractional Fokker-Planck was investigated in the absence of drift. The escape time, or mean first passage time, has also been studied by employing a nonlinear Fokker-Planck equation, leading eventually to a generalization of the Arrhenius law [10]. Other aspects are analysed in 11. All these cases analyse the nonlinear Fokker-Planck equation in one dimension or in $D$-dimension in an isotropic medium. However, a careful analysis of the anisotropic case $\left(\mathcal{D}_{i} \neq \mathcal{D}_{j}\right.$ and $F_{i} \neq F_{j}$, where $\mathcal{D}_{i}$ and $F_{i}$ are the diffusion coefficient and the external force) has not been performed. Thus, it is desirable to study the above results for the nonlinear Fokker-Planck equation in order to cover the anomalous diffusive processes characterized by an anisotropic medium. In this direction, we basically focus our attention on the following anisotropic nonlinear Fokker-Planck equation:

$$
\frac{\partial \rho}{\partial t}=\mathcal{D}_{x} \frac{\partial}{\partial x}\left\{|x|^{-\theta} \frac{\partial \rho^{\nu}}{\partial x}\right\}+\mathcal{D}_{y} \frac{\partial}{\partial y}\left\{|y|^{-\gamma} \frac{\partial \rho^{\nu}}{\partial y}\right\}-\nabla \cdot(\vec{F}(\vec{r}, t) \rho)
$$

where $\vec{F}(\vec{r}, t)$ is the external force $\vec{F}(\vec{r}, t)=\left(-k_{x} x,-k_{y} y\right)$ with $\theta$ and $\gamma \in \mathcal{R}$. It can be verified that $\int_{-\infty}^{\infty} d^{2} r \rho(\vec{r}, t)$ is time independent (hence, if $\rho$ is normalized at $t=0$, it will remain so for ever). Indeed, if we write the equation in the form $\partial_{t} \rho=\nabla \overrightarrow{\mathcal{J}}$ and assume the boundary conditions $\overrightarrow{\mathcal{J}}( \pm \infty, t) \rightarrow 0$, it can be shown that $\int_{-\infty}^{\infty} d^{2} r \rho(\vec{r}, t)$ is a constant of motion. Note that the cases mentioned above [6, 7, 8, 9, 11] can essentially be obtained from Eq. (11) by an adequate choice of the parameters $\theta, \gamma$ and $\nu$. In particular, Eq.(11) reminds us of the equation used to describe diffusion on fractals [12] and recovers, by a suitable choice of $\mathcal{D}_{x}, \mathcal{D}_{y}$ and $\nu$, the special case $\partial_{t} \rho=\overline{\mathcal{D}}_{x} \partial_{x} \partial_{x} \ln (\rho)$, which emerges in plasma physics and in the central limit approximation to Carlemanüs model of the Bolztmann equation[13].

In this work, we first investigate the solutions of Eq. (1) by considering the absence of drift and after we analyze the effect produced by the external force $\vec{F}(\vec{r}, t)=\left(-k_{x} x,-k_{y} y\right)$. In the last situation, the external force is analyzed in two cases, the first one is characterized by $k_{x} / k_{y}=(2+\gamma) /(2+\theta)$ and the second one is given by $k_{x}=k$ and $k_{y}=0$. In addition, we show that the presence of the nonlinearity produces a coupling among the directions, in contrast with the usual Fokker-Planck equation. 


\section{II- ANISOTROPIC NONLINEAR FOKKER-PLANCK EQUATION}

Before discussing the full case, we focus our attention on the free case, i.e., in the absence of drift. Following the approach employed in [6, 8, 14], we can verify that the ansatz

$$
\rho(\vec{r}, t)=\exp _{q}\left[-\beta_{x}|x|^{2+\theta}-\beta_{y}|y|^{2+\gamma}\right] / Z
$$

is the solution of Eq.(11), with $\beta_{x}, \beta_{y}$ and $Z$ being time dependent parameters to be determined and $\nu=2-q$. The $\exp _{q}[x] \equiv[1+(1-q) x]^{\frac{1}{1-q}}$ is the $q$-exponential which arises from the Tsallis formalism 15$]$. It is interesting to mention that some properties of the renormalization group established in [16], such as $\rho(x, t)=\int_{-\infty}^{\infty} d y \rho(\vec{r}, t)$, are verified by Eq.(2) as well as the scaling dependence discussed in [17]. In particular, it is simple to verify that Eq.(2) can be obtained from the Tsallis entropy

$$
S_{q}=\frac{1-\operatorname{Tr} \rho^{q}}{q-1}
$$

by applying the maximum principle of entropy adequated constraints taking into account, $\left\langle\left\langle x^{2+\theta}\right\rangle\right\rangle=\left\langle\left\langle y^{2+\gamma}\right\rangle\right\rangle=$ const. with $\langle\langle\cdots\rangle\rangle=\int d^{2} r[\rho(\vec{r}, t)]^{q}(\cdots) / \int d^{2} r[\rho(\vec{r}, t)]^{q}$ (see also 14]). In order to obtain an explicit form for $\beta_{x}, \beta_{y}$ and $Z(t$ ) we substitute the ansatz given by Eq.(2) into Eq.(1). This replacement leads to the equations:

$$
\begin{aligned}
\frac{1}{Z} \frac{\partial Z}{\partial t} & =\nu(2+\theta) \mathcal{D}_{x} \beta_{x} Z^{1-\nu}+\nu(2+\gamma) \mathcal{D}_{y} \beta_{y} Z^{1-\nu} \\
\frac{1}{\beta_{x}} \frac{\partial \beta_{x}}{\partial t} & =-\nu(2+\theta)^{2} \mathcal{D}_{x} \beta_{x} Z^{1-\nu} \\
\frac{1}{\beta_{y}} \frac{\partial \beta_{y}}{\partial t} & =-\nu(2+\gamma)^{2} \mathcal{D}_{y} \beta_{y} Z^{1-\nu}
\end{aligned}
$$

From the above system of equations we can deduce the relation

$$
\frac{Z(t)}{Z\left(t_{0}\right)}\left(\frac{\beta_{x}(t)}{\beta_{x}\left(t_{0}\right)}\right)^{\frac{1}{2+\theta}}\left(\frac{\beta_{y}(t)}{\beta_{y}\left(t_{0}\right)}\right)^{\frac{1}{2+\gamma}}=1,
$$

where $\beta_{x}\left(t_{0}\right), \beta_{y}\left(t_{0}\right)$ and $Z\left(t_{0}\right)$ are constants. Now, employing Eq.(5) in Eq.(4) and using the relation

$$
\beta_{x}(t)=\left(\frac{2+\gamma}{2+\theta}\right)^{2} \frac{\mathcal{D}_{y}}{\mathcal{D}_{x}} \beta_{y}(t)
$$

we verify that

$$
\beta_{y}(t)=\left\{(1-\varepsilon)(2+\gamma)^{2} \nu \mathcal{D}_{y} \mathcal{C}^{1-\nu} t\right\}^{-\frac{1}{1-\varepsilon}}
$$

where $\mathcal{C}=Z\left(t_{0}\right) \beta_{x}^{\frac{1}{2+\theta}}\left(t_{0}\right) \beta_{y}^{\frac{1}{2+\gamma}}\left(t_{0}\right)\left[(2+\gamma)^{2} \mathcal{D}_{y} /\left((2+\theta)^{2} \mathcal{D}_{x}\right)\right]^{-\frac{1}{2+\theta}}$ and $\varepsilon=(4+\theta+\gamma)(1-\nu) /((2+\theta)(2+\gamma))$. To find $Z(t)$, we may employ Eqs.(5), (6) and (7).

We may obtain further information from the the averages and dispersion relations in order to investigate which combination of $\theta, \gamma$ and $\nu$ leads us to a sub, normal or super diffusion. Thus, for simplicity, we analyse the behavior of $\left\langle x^{2}\right\rangle,\left\langle y^{2}\right\rangle$ and $\left\langle|r|^{2}\right\rangle$. Let us now obtain $\left\langle x^{2}\right\rangle$. By using the definition of average we can show that

$$
\begin{aligned}
\left\langle x^{2}\right\rangle & =\frac{\int d^{2} r x^{2} \rho(\vec{r}, t)}{\int d^{2} r \rho(\vec{r}, t)} \\
& =\frac{1}{\beta_{x}^{\frac{2}{2+\theta}}(t)}\left\{\begin{array}{ll}
\frac{\Gamma\left(\frac{3}{2+\theta}\right) \Gamma\left(\frac{1}{q-1}-\frac{3}{2+\theta}-\frac{1}{2+\gamma}\right)}{(q-1)^{\frac{2}{2+\theta}} \Gamma\left(\frac{1}{2+\theta}\right) \Gamma\left(\frac{1}{q-1}-\frac{1}{2+\theta}-\frac{1}{2+\gamma}\right)} & q>1 \\
\frac{\Gamma\left(\frac{3}{2+\theta}\right) \Gamma\left(\frac{2}{1-q}+\frac{3}{2+\theta}+\frac{1}{2+\gamma}\right)}{(1-q)^{\frac{2}{2+\theta}} \Gamma\left(\frac{1}{2+\theta}\right) \Gamma\left(\frac{2-q}{1-q}+\frac{3}{2+\theta}+\frac{1}{2+\gamma}\right)} & q<1
\end{array} .\right.
\end{aligned}
$$


The behavior of Eq. (8), i.e., sub, normal or super-diffusive, depends on the relation $\theta-((4+\theta+\gamma)(1-\nu)) /(2+\gamma)$ to be less, equal or greater than zero. In the same way, it can be shown that $\left\langle y^{2}\right\rangle \propto 1 / \beta_{y}^{\frac{2}{2+\gamma}}(t)$ and $\left\langle|\vec{r}|^{2}\right\rangle \propto$ $1 / \beta_{x}^{\frac{2}{2+\theta}}(t)+\overline{\mathcal{C}} / \beta_{y}^{\frac{2}{2+\gamma}}(t)$, where $\overline{\mathcal{C}}$ is a constant. In particular, it is interesting to note that $\left\langle|\vec{r}|^{2}\right\rangle$ may exhibit two regimes where the last one presents a diffusion that is faster than the first one, see Fig.(11). Similar behavior for the second moment is found in [18] by considering the anomalous walker diffusion through composite systems.

Now, let us analyse the implications obtained by considering the drift $\vec{F}(\vec{r}, t)=\left(-k_{x} x,-k_{y} y\right)$ with $k_{x} / k_{y}=$ $(2+\gamma) /(2+\theta)$. The drift incorporated in Eq.(1) only leads us to a modifications in (4) altering the time dependence on $\beta_{y}$ as follows:

$$
\beta_{y}=\left\{\frac{(2+\gamma) \nu \mathcal{D}_{y}}{k_{y}} \mathcal{C}^{1-\nu}\left(1-e^{(1-\varepsilon)(2+\gamma) k_{y} t}\right)\right\}^{-\frac{1}{1-\varepsilon}} .
$$

Note that the limit $k_{y} \rightarrow 0$ recovers (7). Let us now consider the case $k_{x}=k$ and $k_{y}=0$, with $\gamma$ and $\theta$ being arbitrary, the equations for the time dependent parameters do not have explicit solutions. In fact, we may only obtain an implicit solution as follows:

$$
\begin{aligned}
& \beta_{x}(t)=\left[\beta_{x}^{\frac{q-3-\theta}{2+\theta}}\left(t_{0}\right) e^{-(1+\theta+\nu) k t}+(1+\theta+\nu)(2+\theta) \nu \mathcal{D}_{x} \mathcal{C}_{1}^{1-\nu} e^{-(1+\theta+\nu) k t} \int_{t_{0}}^{t} d \bar{t} e^{(1+\gamma+\nu) k \bar{t}} \beta_{y}(\bar{t})^{\frac{-1+\nu}{2+\gamma}}\right]^{-\frac{2+\theta}{1+\theta+\nu}} \\
& \beta_{y}(t)=\left[\beta_{y}^{\frac{q-3-\gamma}{2+\gamma}}\left(t_{0}\right)+(1+\gamma+\nu)(2+\gamma) \nu \mathcal{D}_{y} \mathcal{C}_{1}^{1-\nu} \int_{t_{0}}^{t} d \bar{t} \beta_{x}(\bar{t})^{\frac{-1+\nu}{2+\theta}}\right]^{-\frac{2+\gamma}{1+\gamma+\nu}}
\end{aligned}
$$

with $\mathcal{C}_{1}=Z\left(t_{0}\right) \beta_{x}^{\frac{1}{2+\theta}}\left(t_{0}\right) \beta_{y}^{\frac{1}{2+\gamma}}\left(t_{0}\right)$.

From the developments made above we can verify an interesting fact which does not appear in the usual case, the external force applied only in one direction, for example, $x$-direction, manifests itself in all the other directions. This fact may be seen in the time dependence parameters which in this situation are coupling; see the systems of equation (10). In particular, the result that emerges from the above system of equations (10) by considering the second order of the perturbation theory near $t_{0}$ for $\beta_{y}(t)$, as follow:

$$
\begin{aligned}
\beta_{y}(t) & =\left[\beta_{y}^{\frac{q-3-\gamma}{2+\gamma}}\left(t_{0}\right)+(1+\gamma+\nu)(2+\gamma) \nu \mathcal{D}_{y} \mathcal{C}_{1}^{1-\nu} \int_{t_{0}}^{t} d \bar{t}\left(\beta_{x}^{\frac{q-3-\theta}{2+\theta}}\left(t_{0}\right) e^{-(1+\theta+\nu) k\left(\bar{t}-t_{0}\right)}\right.\right. \\
& \left.\left.+\frac{(2+\theta) \mathcal{D}_{x} \nu}{k_{x}} \mathcal{C}_{1}^{1-\nu} \beta_{y}^{\frac{1-q}{2+\gamma}}\left(t_{0}\right)\left(1-e^{-(3+\theta-q) k\left(t-t_{0}\right)}\right)\right)^{\frac{1-\nu}{3+\theta-q}}\right]^{-\frac{2+\gamma}{1+\gamma+\nu}}
\end{aligned}
$$

shows that the external force $\vec{F}=(-k x, 0)$ applied only in the $x$ direction modifies the $y$ direction where we have no drift. In this direction, a simple way to identify, without approximation, the effect of a external force, for the spatial dependence, in an orthogonal direction is to analyse the stationary solution. In particular, for Eq. (10), by considering the drift $\left.\vec{F}(\vec{r}, t)=\left(-\frac{d}{d x} V_{x}(x)\right),-\frac{d}{d y} V_{y}(y)\right)$ and for simplicity $\theta=0$ and $\gamma=0$, the stationary solution is given by

$$
\rho(\vec{r}, t)=\exp _{q}\left[-\beta_{x} V_{x}(x)-\beta_{y} V_{y}(y)\right] / Z_{q},
$$

with $\beta_{y}$ and $\beta_{y}$ obeying the relation $\mathcal{D}_{x} \beta_{x}=\mathcal{D}_{y} \beta_{y}$ and

$$
Z_{q}=\int \exp _{q}\left[-\beta_{x} V_{x}(x)-\beta_{y} V_{y}\right]
$$

\section{III - SUMMARY AND CONCLUSIONS}

In summary, we have worked the nonlinear Fokker-Planck equation in several situations by incorporating an anisotropic dependence in the difusion coefficients and also in the external force. We first analysed the anisotropic case with diffusion coefficients dependent on the position variables and we found its solution, Eq.(2). When we incorporated a drift term with $k_{x} / k_{y}=(2+\gamma) /(2+\theta)$ we verified that Eq.(2) is the solution with a different dependence 
FIG. 1: This figure illustrates the behavior of $\left\langle|\vec{r}|^{2}\right\rangle$ for typical values $\nu, \theta$ and $\gamma$.

on time for the parameters $\beta_{x}, \beta_{y}$ and $Z$. For the case $k_{x}=k$ and $k_{y}=0$, we show that Eq.(2) is also the solution; however, the explicit time dependence on the parameter $Z, \beta_{x}$ and $\beta_{y}$ is very complex leading us to determine them as implicitly. In particular, we have shown that an external force that has only one component when applied to the system governed by Eq.(11) changes the behavior of all directions and consequently the time evolution of the distribution, in contrast with the usual case. The generalization of these results for the multidimensional case is direct when we consider the absence of the drift. In the presence of dritf, we may work the expression in order to recover the above discussion for the bidimensional case. It is important to remark here that the solutions found here can no be written as $\rho(\vec{r}, t)=\rho(x, t) \rho(y, t)$ and an external force applied to the system modifies the behavior of the distribution in all direction, in contrast with the usual case. Finally, we expect that the results obtained here can be useful in the discussion of the anomalous diffusion and its connection with the nonextensive Tsallis thermostatistics.

\section{Acknwonledgements}

We thank CNPq (Brazilian agency) for partial financial support.

[1] M. Muskat, The Flow of Homogeneous Fluid Through Porous Media (McGraw-Hill, New York, 1937).

[2] P. Y. Polubarinova-Kochina, Theory of Ground Water Movement (Princeton University Press, Princeton, 1962).

[3] J. Buckmaster, J. Fluid Mech. 81, 735 (1984).

[4] W. L. Kath, Physica D 12, 375 (1984).

[5] M.F. Shlesinger, G.M. Zaslavsky and U. Frisch, Lévy Flights and Related Topics in Physics (Springer-Verlag, Berlin, 1994).

[6] A. R. Plastino and A. Plastino, Physica A 222, 347 (1995); C. Tsallis and D.J. Bukman, Phys. Rev. E 54, R2197 (1996); L. Borland, Phys. Rev. E 57, 6634 (1998).

[7] G. Drazer, H. S. Wio, C. Tsallis, Phys. Rev. E 61, 1417 (2000).

[8] L.C. Malacarne, I.T. Pedron, R.S. Mendes and E.K. Lenzi, Phys. Rev. E 63, 30101R (2001); I. T. Pedron, R. S. Mendes, L. C. Malacarne and E. K. Lenzi, Phys. Rev. E 65, 41108 (2002).

[9] M. Bologna, C. Tsallis and P. Grigolini, Phys. Rev. E 62, 2213 (2000).

[10] E.K. Lenzi, C. Anteneodo and L. Borland, Phys. Rev. E 63, 051109 (2001).

[11] A. Compte, D. Jou, Y. Katayama, J. Phys. A: Math. Gen. 29, 4321 (1996); L. Borland, F. Pennini, A. R. Plastino and A. Plastino, Eur. Phys. J. B 12, 285 (1999); C. Giordano, A.R. Plastino, M. Casas and A. Plastino, Eur. Phys. J. B 22, 361 (2001); T. D. Frank, J. Math. Phys. 43, 344 (2002).

[12] B. Shaughnessy and I. Procaccia, Phys. Rev. Lett. 54, 455 (1985).

[13] J. G. Berryman and C. J. Holland, J. Math. Phys. 23, 983 (1982); T. G. Kurtz, Tran. Am. Math. Soc. 186, 259 (1973); H. P. McKean, Israel J. Math. 21, 54 (1975).

[14] A. R. Plastino, in S. Abe and Y. Okamoto, Nonextensive Statistical Mechanics and Its Applications, Lecture Notes in Physics (Springer-Verlag, Heidelberg, 2001).

[15] C. Tsallis, J. Stat. Phys. 52, 479 (1988); E. M. F. Curado and C. Tsallis, J. Phys. A 24, L69 (1991); Errata: 24, 3187 (1991); 25, 1019 (1992); C. Tsallis, R. S. Mendes and A. R. Plastino, Physica A 261, (1998) 534; An updated bibliography is accessible at http://tsallis.cat.cbpf.br/biblio.htm

[16] R. S. Mendes and C. Tsallis, Phys. Lett. A 285, 273 (2001).

[17] N. Goldenfeld, Lectures On Phase Transitions and The Renormalization Group, (Addison-Wesley Publishing Company, Inc., Reading, 1992).

[18] C. DeW V. Siclen, J. Phys. A 32, 5763 (1999). 